\title{
Impacto de la trombolisis y de la angioplastia primaria en pacientes con infarto agudo del miocardio tratados en centros hospitalarios terciarios
}

\author{
Douglas G reig ${ }^{1}$, Ramón Corbalán 1 , Pablo Castro ${ }^{1}$, \\ Pabla Campos ${ }^{2}$, Rubén Lamich ${ }^{3}$, Patricio Yovaniniz ${ }^{3}$. \\ Mortality of patients with ST-elevation \\ acute myocardial infarction treated with \\ primary angioplasty or thrombolysis
}

Background: Primary angioplasty is the most effective treatment of ST-segment elevation acute myocardial infarction (STEMI). However, its worldwide implementation is difficult to obtain. Therefore thrombolysis continues to be the treatment most commonly used. Aim: To evaluate in-hospital and long term mortality of patients with STEMI treated with thrombolysis or angioplasty, in three hospitals participating in the Chilean National Registry of Acute MI (GEMI group). Material and methods: Registry of 1,634 consecutive patients with STEMI admited between 2002 and 2006. Risk was stratified using the Thrombolysis in Myocardial Infarction (TIMI) Risk Score. Hospital and log term mortalities were adjusted using logistic and Cox regression models. Results: Fifty nine percent of patients (967 patients aged $60 \pm 12$ years, $77 \%$ males) were subjected to reperfusion therapies, $28 \%$ with primary angioplasty and $72 \%$ with thrombolysis. Hospital mortality rates among patients treated with thrombolysis and angioplasty were $10.9 \%$ and $5.6 \%$ ( $p=0.01$ ), respectively. The figures for long term mortality were $20.4 \%$ and $9.7 \%$, respectively ( $\mathrm{p}<0.01$ ). Multivariate analysis confirmed the lower mortality among subjects treated with angioplasty, with an odds ratio (OR) in favor of angioplasty of 8.5 (95\% confidence intervals (CI) 3-35) for in hospital mortality and of 4.7 (95\% CI 2.6-8.3) for long term mortality. The higher benefits of angioplasty were observed in males, in the elderly and in patients with a TIMI score over >3. Conclusions: Hospital and long term mortality of patients with STEMI was lower among those treated with primary angioplasty. This treatment is most beneficial among males, in the elderly and in patients with a TIMI score >3 (Rev Méd Chile 2008; 136: 1098-106).

(Key words: Angioplasty; Myocardial infarction; Thrombolytic therapy)

Recibido el 21 de enero, 2008. Aceptado el 12 de junio, 2008.

En representación del grupo GEMI y del Departamento de Estudios Multicéntricos, Sociedad Chilena de Cardiología y Cirugía Cardiovascular.

${ }^{1}$ Departamento de Enfermedades Cardiovasculares, Pontificia Universidad Católica de Chile. Santiago, Chile. ${ }^{2}$ Hospital de Urgencias de la Asistencia Pública, Santiago, Chile. ${ }^{3}$ Hospital Barros Luco. Santiago, Chile.

Correspondencia a: Dr. Ramón Corbalán. Departamento de Enfermedades Cardiovasculares, Pontificia Universidad Católica de Chile. Lira $851^{\circ}$ piso, Santiago Centro, Santiago, Chile. E mail: corbalan@med.puc.cl 
L as enfermedades cardiovasculares constituyen la principal causa de mortalidad en la población adulta chilena, siendo el infarto del miocardio (IAM) la mayor causa relativa ${ }^{1}$. La angioplastia primaria (APP) y la trombolisis (TR) constituyen los tratamientos más eficaces para reducir la extensión y mortalidad por infarto del miocardio en las primeras horas de evolución ${ }^{2}$. Se ha demostrado que la APP es más eficaz en términos de éxito en reperfusión coronaria, mayor reducción de la mortalidad y menor incidencia de complicaciones hemorrágicas ${ }^{2}$. Su mayor limitante está dada por la necesidad de tener un equipo médico entrenado y disponible las $24 \mathrm{~h}$ del día y su mayor costo ${ }^{3}$. Esto hace que la TR siga siendo el tratamiento más empleado en todo el mundo y también en nuestro país 4 .

$\mathrm{Si}$ bien ambos tratamientos son ampliamente utilizados en Chile existe información limitada sobre sus eventuales ventajas comparativas y limitaciones en nuestro medio 4 . Por ello hemos analizado la experiencia de 3 centros hospitalarios terciarios que tratan cada año más de 100 pacientes con infarto del miocardio y que por ser parte del Registro GEM ${ }^{5,6}$ han registrado sus datos en una ficha clínica común. Dos de ellos realizaron preferentemente TR y otro exclusivamente APP, en el período analizado.

\section{MÉTOdO}

Población. Se utilizó la base de datos del Registro Nacional del Infarto en Chile (Grupo GEM ${ }^{6}$ ), en el cual se registran todos los ingresos por infarto del miocardio en numerosos centros hospitalarios desde el año 1993 hasta la fecha de acuerdo a una ficha clínica precodificada.

Estudio prospectivo y observacional. Se registraron 2.296 pacientes con IAM consecutivos desde enero de 2001 hasta marzo de 2006 atendidos en 3 centros hospitalarios terciarios de la Región Metropolitana de Santiago de Chile: Hospital Clínico de la Universidad Católica, Hospital de Urgencias de la Asistencia Pública y Hospital Barros Luco. Se seleccionaron estos centros dado que tenían más de 300 ingresos por esta causa y realizaron entre 70 y 100 procedimientos de reperfusión al año durante el período analizado.

Los pacientes fueron incluidos si cumplían los siguientes criterios: 1) síntomas compatibles con
IAM por los menos de 30 min de evolución y no mayores de $6 \mathrm{~h}, 2$ ) cambios ECG en dos derivadas continuas con elevación persistente del segmento $\mathrm{ST} \geq 0,1 \mathrm{mV}$ y 3) elevación de marcadores séricos de necrosis miocárdica mayor a 2 veces el rango normal (troponina T o I, CK total, CK- MB).

Todos los pacientes firmaron el consentimiento informado aprobado por el Comité de Ética de cada institución participante.

Registro de los datos. Los datos fueron ingresados electrónicamente por una enfermera o médico responsable en cada institución. Se registraron datos demográficos, factores de riesgo, antecedentes coronarios previos, características clínicas del IAM (duración del dolor previo a la consulta, localización del IAM, KILUP de ingreso y otros), tratamiento médico al ingreso, fase hospitalaria y al alta, terapia de reperfusión utilizada y tiempo al ser implementada, complicaciones intrahospitalarias, necesidad de nueva coronariografia o cirugía de revascularización, mortalidad intrahospitalaria y a un año de evolución.

Se definió reperfusión exitosa como la presencia de 2 o más de los siguientes criterios no invasivos y de acuerdo a metodología previamente descrita ${ }^{7}$ : a) Descenso del segmento ST $\geq 50 \%$ a los $90 \mathrm{~min}$ de iniciado el procedimiento de reperfusión, b) Elevación precoz de CPK (antes de las $12 \mathrm{~h}$ de iniciada la reperfusión) 0 , c) onda $\mathrm{T}$ negativa en las derivaciones del infarto en las primeras $24 \mathrm{~h}$. Para la estratificación de riesgo del infarto se utilizó el puntaje de riesgo TIMI (TRS) ${ }^{8}$, el que ha sido revalidado en nuestro medio ${ }^{5}$. Todos los análisis fueron realizados en forma centralizada por un observador ajeno y ciego a los centros hospitalarios involucrados.

Seguimiento. Para los efectos de mortalidad alejada y sus causas se utilizó la base de datos de la Oficina de Registro Civil e Identificación. En todos los casos en que la información acerca de la causa de muerte no fue clara, se realizó confirmación telefónica. El seguimiento en cuanto a mortalidad fue de $100 \%$, mientras que se logró establecer fidedignamente la causa de ésta en 98\%. El promedio de seguimiento fue de 859 días (30 a 1.944 días). Para efectos del cálculo del porcentaje de mortalidad, se consideraron los eventos ocurridos hasta un año del seguimiento. 
Estadística. Los datos son presentados como promedio \pm desviación estándar. Los grupos fueron comparados mediante test de $\mathrm{t}$ de student para variables independientes o mediante test de chicuadrado, para variables continuas o categóricas según corresponda. Para el análisis de la mortalidad intrahospitalaria (MIH) se realizaron modelos de regresión logística. La mortalidad alejada (MA) fue comparada mediante curvas de Kaplan Meier y log rank. Se elaboraron modelos de regresión de Cox. Todos los modelos fueron ajustados en base a los predictores independientes de mortalidad por IAM en nuestro medio: edad, género, diabetes, localización anterior y Killip $\geq 1^{9}$ y por el TRS.

Para establecer la edad de corte con la mayor significancia se incluyeron en el modelo de regresión múltiple los diferentes cuartiles de edad en forma independiente, la cual fue ajustada por sexo y método de reperfusión. Un valor $\mathrm{p} \leq 0,05$ fue considerado significativo. Todos los análisis fueron realizados con SPSS 15.0.

\section{RESULTADOS}

Características de los pacientes. De los 2.296 pacientes registrados inicialmente en el estudio, 1.634 (71\%) correspondieron a IAM con supradesnivel del segmento ST. De éstos, 967 (59,2\%) fueron tratados con terapias de reperfusión, 72\% con TR (90\% con estreptoquinasa y 10\% con trombolíticos específicos) y $28 \%$ con APP, los que constituyen la base de nuestro estudio. En el grupo que no recibió terapias de reperfusión, las principales causas fueron: ingreso tardío (46\%), el no presentar dolor al ingreso (16\%) y contraindicaciones formales al uso de trombolíticos (10\%). Las características basales de los pacientes se muestran en la Tabla 1. La edad promedio fue de $60 \pm 12$ años, 77\% de sexo masculino. Los pacientes sometidos a APP tenían un mayor porcentaje de dislipidemia, tabaquismo, procedimientos de revascularización previos y mayor grado de KILUP al ingreso (Tablas 1 y 2). El tiempo comprendido entre el ingreso al hospital e inicio de la terapia de reperfusión (tiempo puerta-aguja o puerta-balón, según corresponda) fue significativamente mayor en los pacientes sometidos a APP, pese a lo cual el porcentaje de reperfusión exitosa fue mayor en este grupo (Tabla 2). Con respecto a la APP, el promedio de vasos culpables fue de $1,7 \pm 0,7$, en $86 \%$ de los casos se utilizó 1 stent, siendo $60 \%$ stent metálico, y en $32 \%$ stent con droga. Se obtuvo flujo TIMI III en $90 \%$ de los casos.

Efecto del método de reperfusión y otras variables en la mortalidad intrahospitalaria y alejada. La MH global en TR y APP fue de 10,9 vs $5,6 \%$ (p $<0,001$ ) y la MA fue de 20,4 vs $9,7 \%$ ( $p<0,001$ ), respectivamente (Figura 1). La Tabla 3 muestra

Tabla 1. Características generales de los pacientes según método de reperfusión

\begin{tabular}{|c|c|c|c|}
\hline & $\begin{array}{c}\text { APP } \\
(n=271)\end{array}$ & $\begin{array}{c}\text { TR } \\
(n=696)\end{array}$ & Valor $\mathrm{p}$ \\
\hline Sexo masculino (\%) & 79,3 & 76,3 & 0,4 \\
\hline Edad (promedio \pm DE, años) & $59,7 \pm 14$ & $59,5 \pm 11$ & 0,8 \\
\hline HTA $(\%)$ & 50 & 51,1 & 0,8 \\
\hline Diabetes mellitus (\%) & 18,5 & 24,4 & 0,06 \\
\hline Dislipidemia (\%) & 45,9 & 28,6 & 0,001 \\
\hline Tabaquismo (\%) & 96,2 & 78,3 & 0,001 \\
\hline IAM previo (\%) & 8,5 & 10,6 & 0,4 \\
\hline \multicolumn{4}{|l|}{ Revascularización previa (\%) } \\
\hline PTCA & 5,6 & 3,3 & 0,14 \\
\hline CRM & 2,6 & 1,1 & 0,14 \\
\hline Angina crónica (\%) & 3,3 & 10 & 0,001 \\
\hline
\end{tabular}


Tabla 2. C aracterísticas del infarto según método de reperfusión utilizado

\begin{tabular}{|llll|}
\hline & APP & TR & Valor $\mathrm{p}$ \\
\hline KILLP $>1(\%)$ & 21,8 & 15 & 0,02 \\
TRS $\geq 3(\%)$ & 50,4 & 48,8 & 0,67 \\
Tiempo a tratamiento (min \pm DE) & $59 \pm 38$ & $41 \pm 45$ & $<0,001$ \\
Localización anterior (\%) & 49,2 & 49,8 & 0,82 \\
Reperfusión exitosa (\%) & 80,7 & 65,9 & $<0,001$ \\
\hline
\end{tabular}

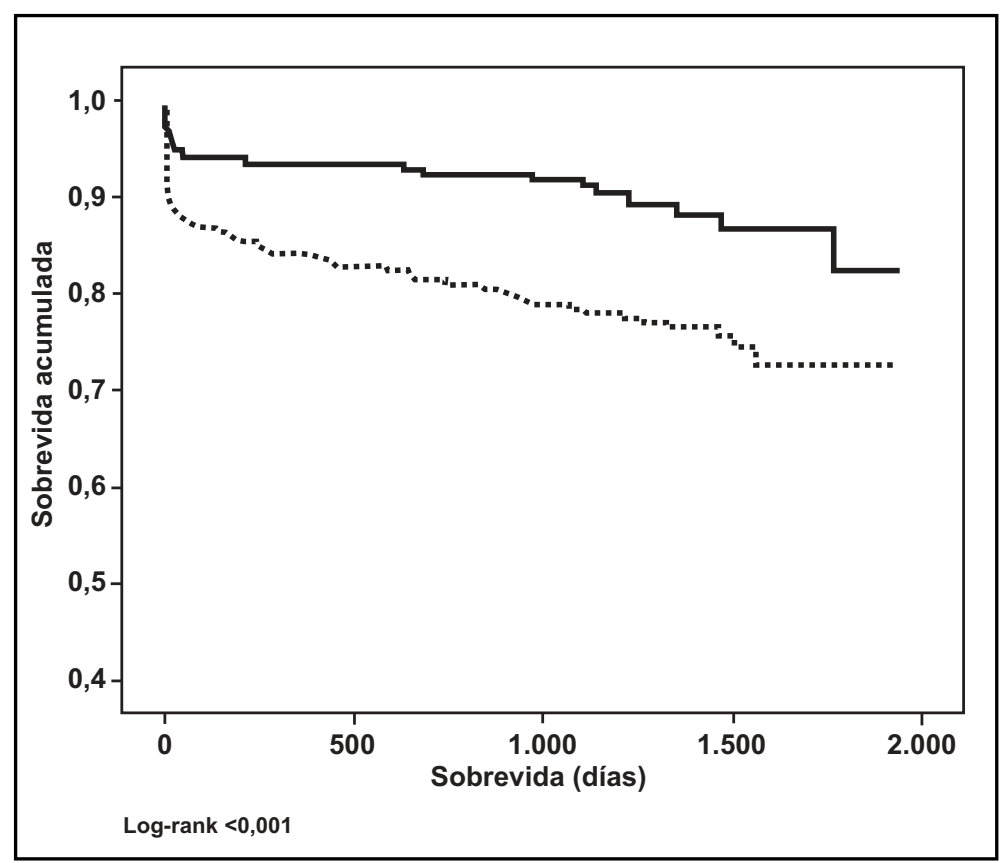

Figura 1. Curva de sobrevida según método de reperfusión (línea continua: APP; Línea discontinua: TR).

\section{Tabla 3. M ortalidad intrahospitalaria y alejada, ajustada según método de reperfusión y características del infarto}

\begin{tabular}{|lll|}
\hline & $\begin{array}{l}\text { Mortalidad intrahospitalaria } \\
\text { OR (IC 95\%) }\end{array}$ & $\begin{array}{l}\text { Mortalidad alejada } \\
\text { HR (IC 95\%) }\end{array}$ \\
\hline Trombolisis vs angioplastia & $6,95(2,5-19,3)^{\dagger}$ & $4,0(2,2-7,1)^{\dagger}$ \\
Edad $>70$ años & $4,0(2,2-7,6)^{\dagger}$ & $3,6(2,5-5,2)^{\dagger}$ \\
Sexo femenino & $1,5(0,8-2,9)^{\&}$ & $1,4(0,7-2,1)^{\&}$ \\
KILUP $>2$ & $9,2(3,7-22,6)^{\dagger}$ & $4,5(2,7-7,7)^{\dagger}$ \\
Glicemia $>140 \mathrm{mg} / \mathrm{dl}$ & $3,0(1,5-6,0)^{\dagger}$ & $1,8(1,2-2,8)^{\dagger}$ \\
\hline
\end{tabular}
$\dagger_{:} \mathrm{p}<0,001$
$\&: p=n s$ 
aquellas variables significativas que influyen en la MHH y MA en el modelo multivariado. Se observa que las variables que más influyen son la TR comparado con la APP, seguido del KILUP de ingreso y la edad. Del mismo modo, se observa un mayor beneficio de la APP sobre la TR en la $\mathrm{MH}$ en los pacientes mayores de 60 años (Figura 2). Al comparar las diferencias según sexo, la MH y MA fue mayor en el sexo femenino comparado con el sexo masculino tanto en el subgrupo tratado con APP como con TR (Figura 3). Al comparar la MA según método de reperfusión separados por sexo, se observa un mayor beneficio de la APP sobre la TR en los pacientes de sexo masculino comparado al sexo femenino ( $\mathrm{HR}=8,4$ IC $95 \%=3,3-21,1$ vs $\mathrm{HR}=2,3$ IC $95 \%=1-5,5$, respectivamente). Pese a lo anterior, el sexo femenino no confirió un mayor riesgo en los modelos multivariados considerando el grupo global (Tabla 3). El porcentaje de pacientes con reperfusión exitosa según criterios no invasivos fue también significativamente mayor en los pacientes tratados con APP.

Por otro lado, al ajustar la MA por puntaje de riesgo TIMI se observa que el beneficio de la APP es mayor en aquellos pacientes con puntaje $\geq 3$ puntos (Figura 4).

La Tabla 4 muestra la MH y MA ajustada por método de reperfusión y uso de betabloquedores, estatinas o inhibidores de la enzima convertidora de angiotensina II (IECA) o bloqueadores del receptor de angiotensina II (ARA II) en aquellos pacientes que no las estaban empleando previo a su ingreso. Según se observa, las tres terapias disminuyeron en forma significativa, tanto la MIH como la MA, independiente del método de reperfusión empleado.

La Tabla 5 muestra la distribución de la terapia farmacológica intrahospitalaria y al alta según el tipo de reperfusión. Se observa que los pacientes sometidos a APP tuvieron una mejor terapia farmacológica, dado por un mayor porcentaje de betabloqueadores, estatinas y antagonistas de la aldosterona. Sin

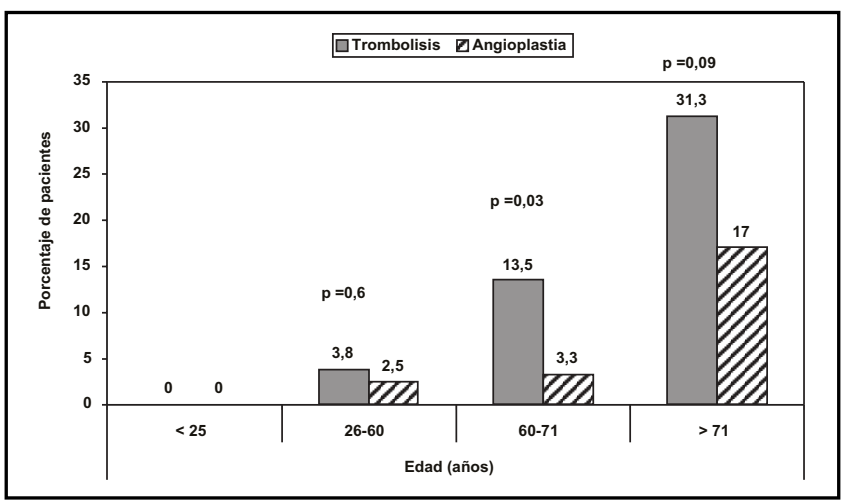

Figura 2. Mortalidad intrahospitalaria según edad y método de reperfusión.

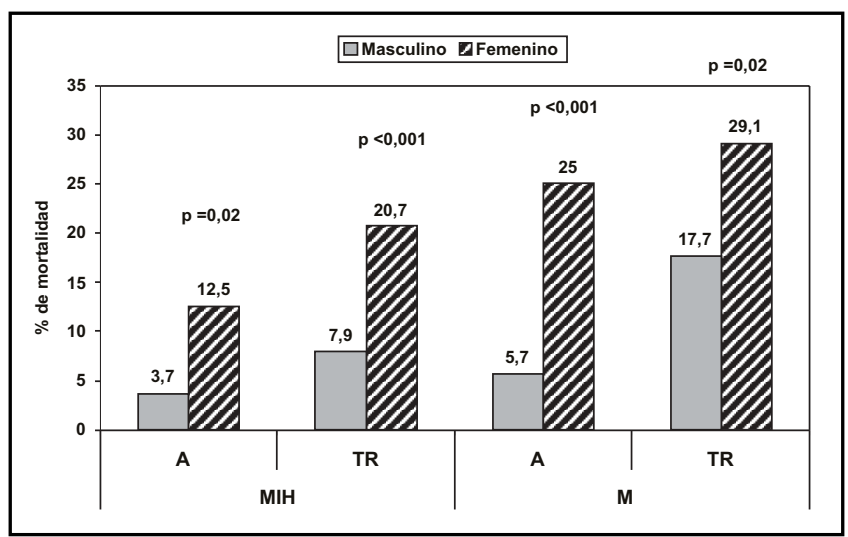

Figura 3. Mortalidad intrahospitalaria y alejada según sexo y método de reperfusión.

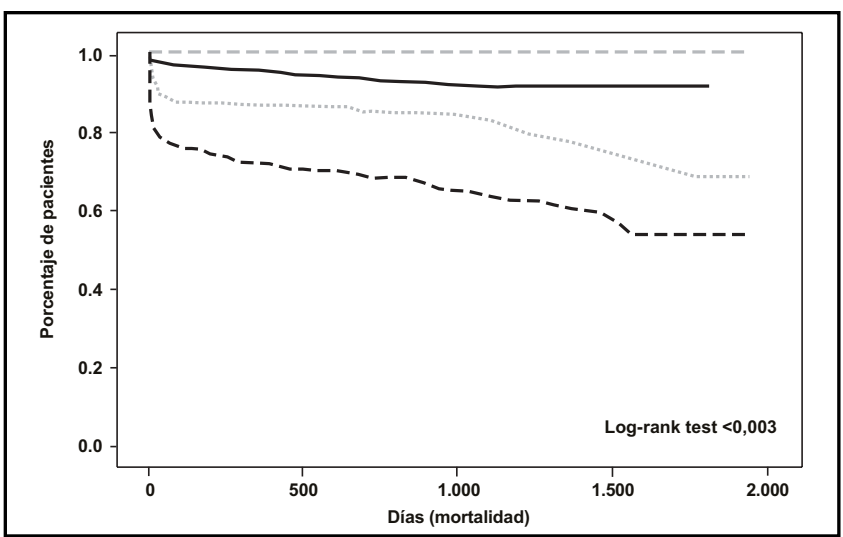

Figura 4. Mortalidad alejada según método de reperfusión y TRS. ( - - -: APP y TRS $<3$ puntos; __ : TR y TRS $<3$ puntos; : APP y TRS $\geq 3$ puntos; - - - - : TR y TRS $\geq 3$ puntos). 
Tabla 4. Efecto de la terapia médica concomitante en la mortalidad intrahospitalaria y alejada, ajustada por características del IAM y método de reperfusión

\begin{tabular}{|c|c|c|}
\hline & $\begin{array}{c}\text { Mortalidad intrahospitalaria } \\
\text { OR (IC 95\%) }\end{array}$ & $\begin{array}{c}\text { Mortalidad alejada } \\
\text { HR (IC 95\%) }\end{array}$ \\
\hline Betabloqueadores & $0,2 \quad(0,1-0,4)$ & $0,5 \quad(0,3-0,7)$ \\
\hline IECA o ARA II & $0,19(0,09-0,4)$ & $0,5 \quad(0,3-0,7)$ \\
\hline Estatinas & $0,19(0,1-0,4)$ & $0,4 \quad(0,3-0,6)$ \\
\hline
\end{tabular}

IECA: Inhibidores de la enzima convertidora de angiotensina. ARA II: Inhibidores de los receptores de angiotensina II.

Tabla 5. M edicamentos indicados durante el periodo intrahospitalario y al alta, según el método de reperfusión empleado

\begin{tabular}{|lllll|}
\hline & & APP & TR & Valor p \\
\hline \multirow{2}{*}{ Aspirina } & IH & 98,5 & 99 & 0,5 \\
& Alta & 82,1 & 79,2 & 0,09 \\
Clopidogrel & IH & 89,3 & 24 & 0,001 \\
Betabloqueadores & Alta & 79,2 & 23,5 & 0,001 \\
IECA o ARA II. & IH & 85 & 73 & 0,001 \\
\multirow{2}{*}{ Antagonistas de aldosterona. } & Alta & 76 & 64 & 0,001 \\
\multirow{2}{*}{ Estatinas } & IH & 60,7 & 75,6 & 0,001 \\
& Alta & 50,4 & 64,1 & 0,001 \\
& IH & 12,2 & 4,9 & 0,001 \\
& Alta & 9,3 & 4,2 & 0,03 \\
& IH & 74,4 & 58,8 & 0,001 \\
\hline
\end{tabular}

IH: Terapia intrahospitalaria; Alta: terapia prescrita en el egreso hospitalario

embargo, los mismos pacientes recibieron un menor porcentaje de IECA o ARA II. La terapia antiagregante plaquetaria fue comparable, considerando el mayor uso de clopidogrel en el grupo tratado con APP.

\section{DISCUSIÓN}

Nuestro estudio mostró que la mayoría de los pacientes ingresados a los centros seleccionados reciben alguna terapia de reperfusión, siendo en la mayoría de los casos la TR con estreptoquinasa. Sin embargo, la proporción de pacientes que no recibieron terapias de reperfusión fue mayor a la descrita en otros registros internacionales ${ }^{10}$, pero concordante con datos globales del registro GEMI, previo a la implementación del protocolo AUGE5,6.

Actualmente, la APP se considera la terapia más recomendable en el tratamiento de pacientes con IAM con SDST ${ }^{2,11}$. Al igual que en otros reportes, la mortalidad con APP sigue siendo menor comparada con TR, pese a que los pacientes sometidos a APP son habitualmente más graves según grado de Killip al ingreso o tienen más factores de riesgo ${ }^{2,11,12}$, como fue el caso en nuestro estudio. Por esta razón en los registros de países desarrollados se observa un empleo cada vez más alto de APP en reemplazo de la $\mathrm{TR}^{13,14}$. 
Esta tendencia se está observando también en nuestro país donde se ha ido incorporando cada vez más la APP en hospitales públicos y privados. De hecho el Hospital Barros Luco, incluido en este análisis, ha derivado también a la implementación de APP para el manejo del IAM en los últimos 2 años. Por otra parte es interesante observar que los pacientes tratados con TR tenían más antecedentes de angina crónica y menores antecedentes de angioplastia o cirugía de revascularización coronaria. Esto podría reflejar las terapias más agresivas en los centros en los que se realizan APP a diferencia de los centros en que se realiza TR en que el acceso a estudios invasivos era menor en el período analizado.

Por otro lado, nuestro estudio valida una vez más la importancia del puntaje de riesgo TIMI, el cual fue derivado a partir de una población de pacientes con IAM y SDST tratados con $\mathrm{TR}^{8}$. Este puntaje de riesgo incluye un total de 14 variables relacionadas con la gravedad del IAM, entre ellas edad avanzada, insuficiencia cardiaca, antecedentes previos y otras.

La mayor mortalidad en las mujeres en nuestro estudio es concordante con lo reportado en la literatura ${ }^{15,16}$ y en análisis previos del grupo $\mathrm{GEM}^{6}$. Diversos autores han postulado que esto podría deberse a que las mujeres tienen mayor riesgo ${ }^{15,17}$. En nuestro registro, las mujeres presentaron un puntaje de riesgo más alto, eran de mayor edad y recibieron una terapia farmacológica menos óptima (datos no mostrados). Pese a esto, al igual que lo reportado en otros registros, el riesgo de MA en las mujeres ajustado por estas características y por método de reperfusión no fue mayor que en los hombres en este análisis, diferencia que podría deberse a una casuística de menor tamaño ${ }^{15,16,18,19}$. En los hombres, se observó un claro beneficio de la APP sobre la TR, situación que no alcanzó diferencia estadística en las mujeres. Una posible explicación de este beneficio en los hombres, podría ser explicado también por el diferente perfil de riesgo de las mujeres y por el bajo número de mujeres tratadas.

Algunos reportes han señalado que, en parte, el beneficio de la APP está dado por el mejor tratamiento farmacológico en este subgrupo ${ }^{20,21}$. En nuestro estudio, encontramos diferencias significativas en la prescripción de medicamentos intrahospitalarios y al egreso en las 2 poblaciones analizadas. Sin embargo, el beneficio de la APP sobre la TR se mantuvo luego de ajustar según la terapia farmacológica coadyuvante. Además, en nuestro estudio se observó que el beneficio sobre la mortalidad de betabloqueadores, IECA, ARA II y estatinas, es independiente del tipo de terapia de reperfusión utilizada. Recientemente, Fox y $\operatorname{cols}^{22}$ demostraron que los cambios en la prescripción farmacológica en dicho período (años 1999 a 2006) en el registro GRACE se asociaron a una disminución de mortalidad. Si bien nosotros no registramos una diferencia en la prescripción de terapias farmacológicas en el periodo estudiado (datos no mostrados), resultó también evidente el beneficio de la terapia farmacológica mencionada y cuyo empleo fue superior al reportado previamente en nuestro país en la década pasada ${ }^{23}$.

La menor mortalidad observada en los pacientes tratados con APP versus TR se logró a pesar de que el tiempo promedio entre el diagnóstico y el inicio de tratamiento fue mayor en los pacientes del grupo APP. Un estudio reciente del Registro Nacional de IAM de Estados Unidos de Norteamérica demuestra que el tiempo entre inicio de terapia con balón no debe exceder en $60 \mathrm{~min}$ al tiempo transcurrido entre el diagnóstico e inicio de trombolisis ${ }^{24}$ para obtener un impacto sobre la mortalidad. Tal fue el caso en este estudio en que el tiempo promedio al inicio de APP fue de $58 \mathrm{~min}$ versus 48 min en los casos tratados con TR. Estas observaciones son pertinentes para los grupos de trabajo que se están incorporando al manejo del IAM con APP, los que además de tener experiencia con esta técnica deben optimizar los tiempos de tratamiento.

Los porcentajes de reperfusión exitosa alcanzados con APP y TR $(80,7$ v 65,9\%) son concordantes con reportes previos de nuestro grupo ${ }^{6,24}$. Independientemente del alto porcentaje de flujo TIMI 3 que se obtiene con la APP (90\%) es conocido que un porcentaje variable de estos pacientes no obtiene una reperfusión exitosa a nivel microvascular, lo que se asocia a un pronóstico más adverso 24 .

Los actuales registros internacionales de pacientes con infarto agudo del miocardio con SDST no representan necesariamente la realidad de nuestros centros hospitalarios terciarios ${ }^{10,14,19,26}$. Una de las limitaciones de nuestro análisis es que la información obtenida proviene de sólo 3 de los 
centros hospitalarios del registro GEMI, pero se escogieron por ser los centros que históricamente han aportado más pacientes al mismo y en el período analizado utilizaron una estrategia de reperfusión constante. Los resultados obtenidos nos permiten caracterizar mejor nuestra población y precisar en qué tipo de pacientes se obtiene mayores beneficios de la APP, lo que sería de utilidad en un futuro próximo para establecer redes de derivación de pacientes a centros con

\section{REFERENCIAS}

1. Departamento de Estadísticas e Información en Salud. Ministerio de Salud de Chile 2007; URL: http/ /deis.minsal.cl/index.asp.

2. Keeley EC, Boura JA, Grines CL. Primary angioplasty versus intravenous thrombolytic therapy for acute myocardial infarction: a quantitative review of 23 randomized trials. Lancet 2003; 361: 13-20.

3. Nalamothu B, Fox KA, Kenneluy BM, Van de WF, Gore JM, Steg PG et al. Relationship of treatment delays and mortality in patients undergoing fibrinolysis and primary percutaneous coronary intervention. The Global Registry of Acute Coronary Events. Heart 2007; 93: 1552-5.

4. Chamorro H, Ducci H, Mathei R, Alcaíno M, FlorenzaNo F, RAmírez A ET AL. Angioplastia coronaria primaria como tratamiento de elección en las primeras 6 horas del infarto de miocardio. Rev Méd Chile 1995; 123: 727-34.

5. Corbalán R, Nazzal C, Prieto JC, Chávez E, lanas F, LAMICH R ET al. Reducción de la mortalidad por infarto del miocardio en hospitales chilenos. Rev Méd Chile 2002; 130: 368-78.

6. Prieto JC, Corbalán R, Chávez E, Lanas F, Cumsile F, NazzaL C. Infarto agudo del miocardio en hospitales chilenos: resultados finales del estudio GEMI. Rev Méd Chile 1999; 127: 763-74.

7. Corbalán R, Prieto JC, Chávez, MD, Nazzal C, Cumsile F, KRUCOFF M. Bedside markers of coronary artery patency and short-term prognosis of patients with acute myocandial infarction and thrombolysis. Am Heart J 1999; 138: 533-9.

8. Morrow DA, Antman EM, Charlesworth A, Cairns R, Murphy SA, DE Lemos JA ET AL. TIMI risk score for STelevation myocardial infarction: A convenient, bedside, clinical score for risk assessment at presentation: An intravenous nPA for treatment of infarcting myocardium early II trial substudy. Circulation 2000; 102: 2031-7. experiencia en esta técnica. La reciente implementación del protocolo AUGE para el manejo del IAM en nuestro país está resultando en un mayor empleo de trombolíticos y fármacos basados en evidencia y el financiamiento de la APP en hospitales públicos está derivando a un empleo cada vez mayor de esta estrategia terapéutica, que como se demuestra en el presente estudio tiene claros beneficios en los pacientes con IAM y perfil de riesgo más alto.

9. DeGeare VS, Boura Ja, Grines LL, O'Neill WW, Grines CL Predictive value of the Killip classification in patients undergoing primary percutaneous comnary intervention for acute myocardial infarction. Am J Cardiol 2001; 87: 1035-8.

10. GiBson CM. NRMI and current treatment patterns for ST-elevation myocardial infarction. Am Heart J 2004; 148: S29-S33.

11. Weaver WD, Simes RJ, Betriu A, Grines CL, Zijlstra F, GarCí E ET AL. Comparison of primary coronary angioplasty and intravenous thrombolytic therapy for acute myocardial infarction: a quantitative review. JAMA 1997; 278: 2093-8.

12. Boersma E. Does time matter? A pooled analysis of randomized clinical trials comparing primary percutaneous coronary intervention and in-hospital fibrinolysis in acute myocardial infarction patients. Eur Heart J 2006; 27: 779-88.

13. Bjorklund E, Lindahl B, Stenestrand U, Swahn E, Deluborg M, Pehrsson K et al. Outcome of STelevation myocardial infarction treated with thrombolysis in the unselected population is vastly different from samples of eligible patients in a largescale clinical trial. Am Heart J 2004; 148: 566-73.

14. Rogers WJ, Canto JG, Lambrew CT, Tiefenbrunn AJ, KinKaID B, Shoultz DA et aL. Temporal trends in the treatment of over 1.5 million patients with myocardial infarction in the US from 1990 through 1999: the National Registry of Myocardial Infarction 1, 2 and 3. J Am Coll Cardiol 2000; 36: 2056-63.

15. De LG, Suryapranata H, Dambrink JH, Ottervanger JP, VAN'T Hof AW, ZIJLSTRA F ET AL. Sex-related differences in outcome after ST-segment elevation myocardial infarction treated by primary angioplasty: data from the Zwolle Myocardial Infarction Study. Am Heart J 2004; 148: 852-6.

16. Kosuge M, Kimura K, Kojima S, Sakamoto T, Ishihara M, ASADA Y ET AL. Sex differences in early mortality of patients undergoing primary stenting for acute myocardial infarction. Circ J 2006; 70: 217-21. 
17. Mahon NG, McKenna CJ, Codd MB, O’Rorke C, MCCANN HA, Sugrue DD. Gender differences in the management and outcome of acute myocardial infarction in unselected patients in the thrombolytic era. Am J Cardiol 2000; 85: 921-6.

18. Malacrida R, Genoni M, Maggioni AP, Spataro V, Parish S, Palmer A ET al. A comparison of the early outcome of acute myocardial infarction in women and men. The Third International Study of Infarct Survival Collaborative Group. N Engl J Med 1998; 338: 8-14.

19. Vaccarino V, Parsons L, Every NR, Barron HV, KRUMHOLz HM. Sex-based differences in early mortality after myocardial infarction. National Registry of Myocardial Infarction 2 Participants. N Engl J Med 1999; 341: 217-25.

20. Widimsky P, Zelizko M, Jansky $P$, Tousek $F$, Holm $F$, Aschermann M. The incidence, treatment strategies and outcomes of acute coronary syndromes in the "reperfusion network" of different hospital types in the Czech Republic: results of the Czech evaluation of acute coronary syndromes in hospitalized patients (CZECH) registry. Int J Cardiol 2007; 119: 212-9.

21. Anderson JL, Karagounis LA, Muhlestein JB. Explaining discrepant mortality results between primary percutaneous transluminal coronary angioplasty and thrombolysis for acute myocardial infarction. Am J Cardiol 1996; 78: 934-9.
22. Fox KA, Steg PG, Eagle KA, Goodman SG, Anderson FA JR., Granger CB ET AL. Decline in rates of death and heart failure in acute coronary syndromes, 19992006. JAMA 2007; 297: 1892-900.

23. Prieto JC, Corbalán R, Nazza C, Chávez E, Lanas F, Bartolucci J, Cumsile F. Cambios en los patrones de prescripción de medicamentos en el infarto agudo del miocardio. Comparación de dos períodos. Rev Méd Chile 2001; 129: 481-8.

24. Corbalán R, Larraín G, Nazzal G, Castro P, Acevedo $\mathrm{M}$, Domínguez JM et aL. Association of noninvasive markers of coronary artery reperfusion to assess microvascular obstruction in patients with acute myocardial infarction treated with primary angioplasty. Am J Cardiol 2001; 88: 342-6.

25. Pinto DS, Kirtane AJ, Nalamothu BK, Murphy SA, Cohen DJ, Laham RJ ET AL. Hospital delays in reperfusion for ST-elevation myocardial infarction: implications when selecting a reperfusion strategy. Circulation 2006; 114: 2019-25.

26. Randomized trial of intravenous streptokinase, oral aspirin, both, or neither among 17,187 cases of suspected acute myocardial infarction: ISIS-2. ISIS-2 (Second International Study of Infarct Survival) Collaborative Group. Lancet 1988; 2: 349-60.

27. Steg PG, López-Sendon J, López de SE, Goodman SG, Gore JM, ANDERSON FA ET AL. External validity of clinical trials in acute myocardial infarction. Arch Intern Med 2007; 167: 68-73. 